\title{
\#Epi: There is no place for the use of intravenous epinephrine as a standard component of cardiac arrest resuscitation care
}

\author{
Michelle Welsford, BSc, MD*+; Jason E. Buick, MSc, ACPf ; Ian R. Drennan, ACP§ף; Steve Lin, MD,

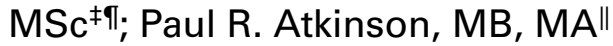

\section{INTRODUCTION}

\section{Paul Atkinson (@eccucourse)}

We continue the \#CFEMDebate series with the topic of whether epinephrine (adrenalin) should continue to be routinely administered during the management of sudden cardiac arrest (SCA), a debate that is particularly relevant following recent publications and the resulting conversations within emergency medical service (EMS) and emergency medicine circles. Are we causing more harm than good with continued routine use of epinephrine during SCA? Or must we use every tool that shows any hope of improving outcomes in this patient group, in whom the failure of therapy is final? Does the latest evidence support current protocols or even modified use of epinephrine, or do we persist in using a treatment option that is based purely on physiological models and expert opinion, without a strong evidence base? Further, from a cost perspective, is epinephrine use providing meaningful health improvements for society, or is it a cost without any benefits?

Arguing that epinephrine has had its day are Dr. Michelle Welsford, a paramedic wannabe but also an emergency physician at Hamilton Health Sciences, the Medical Director for the Centre for Paramedic Education \& Research, and a Professor and Division
Director of Emergency Medicine at McMaster University, who has been an evidence-based nerd and involved with resuscitation guideline creation for more years than she wants to admit, along with Jason Buick, an advanced care paramedic in the Greater Toronto area and a PhD student at the Institute of Health Policy, Management and Evaluation (IHPME) at the University of Toronto, who researches clinical prediction rules for paramedics and prehospital resuscitation of lifethreatening emergencies. In response, supporting another round of epinephrine, are Ian Drennan, an advanced care paramedic with York Region Paramedic Services and a $\mathrm{PhD}$ candidate at the University of Toronto and St. Michael's Hospital, who researches cardiac resuscitation and the treatment of other lifethreatening illness and injury in the prehospital setting, along with Dr. Steve Lin, an Emergency Physician, Trauma Team Leader, and Scientist at St. Michael's Hospital and Assistant Professor in the Department of Medicine and the IHPME at the University of Toronto, whose translational research program aims to optimize cardiac arrest resuscitation. This experienced team are all actively engaged in resuscitation outcomes research with organizations such as the International Liaison Committee on Resuscitation (ILCOR) and the Canadian Resuscitation Outcomes Consortium (CanROC).

From *Division of Emergency Medicine, McMaster University, Hamilton, ON; tCentre for Paramedic Education \& Research, Hamilton Health Sciences, Hamilton, ON; ¥Institute of Health Policy, Management and Evaluation, University of Toronto, Toronto, ON; §Institute of Medical Sciences, Faculty of Medicine, University of Toronto, Toronto, ON; ๆLi Ka Shing Knowledge Institute, St. Michael's Hospital, Toronto, ON; and the ॥Department of Emergency Medicine, Dalhousie University, Saint John Regional Hospital, Saint John, NB.

Correspondence to: Dr. Paul Atkinson, Department of Emergency Medicine, Dalhousie University, Saint John Regional Hospital, 400 University Ave., Saint John, NB, E2L 4L4; Email: paul.atkinson@ dal.ca

(c) Canadian Association of Emergency Physicians

CJEM 2019;21(3):324-329

DOI 10.1017/cem.2019.17 
Readers can follow the debate on Twitter and vote for either perspective, by going to @CFEMonline or searching \#CFEMDebate.

FOR THE MOTION: EPINEPHRINE; ONLY PROVEN BENEFICIAL FOR DOGS?

\section{Michelle Welsford (@WelsfordM) and Jason E. Buick (@jason_buick)}

\section{What interventions in cardiac arrest have shown to improve good neurological survival?}

Immediate bystander intervention, high-quality cardiopulmonary resuscitation (CPR), and early defibrillation save lives! ${ }^{1}$ Epinephrine? Not so fast.

\section{Wasn't there strong evidence for epinephrine when it was introduced?}

Epinephrine was suggested for cardiac arrest in the early 1960s by Dr. Peter Safar, the "father of CPR" at doses of $10 \mathrm{mg}$ intravenously (IV). ${ }^{2}$ Animal experiments showed that $1 \mathrm{mg}$ of epinephrine (high dose of $25 \mathrm{mg} / \mathrm{kg}$ ) intracardiac or through the femoral vein resulted in the greater return of spontaneous circulation (ROSC) and survival to 24 hours, without evidence of long-term survival. ${ }^{3}$ It is time to reconsider our current cardiac arrest management that was based upon 60-year-old data from studies in dogs.

\section{What are the physiologic benefits and detriments of epinephrine?}

The potentially beneficial effects of epinephrine in cardiac arrest are primarily the alpha effects: peripheral vasoconstriction and augmentation of aortic diastolic pressure, which results in increased coronary and cerebral blood flow. ${ }^{4,5}$ Some of the beta effects such as increased heart rate and force of contraction may be beneficial as well. ${ }^{4}$

However, many of the beta effects of epinephrine are likely to be harmful, causing increased myocardial oxygen demand, cardiac irritability and dysrhythmias, decreased cerebral microcirculation, and circulatory mismatch in the lungs. The effects of excessive beta stimulation appear to be particularly detrimental in ventricular fibrillation arrests. This may lead to greater post-ROSC tissue hypoperfusion, cardiac re-arrest, and severe neurological impairment. ${ }^{4}$
What about evidence for epinephrine dosing in adults?

In the 1990s, high-dose epinephrine (2-10 mg IV) was evaluated in adults in several randomized controlled trials (RCT) and found that although high-dose epinephrine was associated with ROSC, it showed no benefit in survival to discharge. ${ }^{6,7}$ Additionally, the use of high-dose epinephrine in children showed improved ROSC, but this failed to translate to increased survival to discharge, so the use of high-dose epinephrine was abandoned. ${ }^{8}$ There are no controlled human studies on using doses of epinephrine lower than $1 \mathrm{mg}$ every 3-5 minutes, despite this dose being based upon $15-30 \mathrm{~kg}$ dogs.

Has anyone studied the use of epinephrine compared with placebo in humans?

In the last decade, several large observational studies have shown an adjusted association of epinephrine with ROSC and survival to admission but have also found an association with reduced survival to discharge and good neurological survival (Cerebral Performance Category $1-2){ }^{9}$

In the last decade, three RCTs have been published comparing epinephrine with placebo (or no epinephrine) in out-of-hospital cardiac arrest (OHCA). ${ }^{10-12}$ The first of these studies randomized 851 patients in Norway to IV cannulation with medications (both epinephrine and antiarrhythmics) compared with no IV or medications. ${ }^{11}$ The second study randomized 534 patients in Australia to epinephrine or placebo, but the study was stopped after recruitment of only $10 \%$ of the intended sample size related to difficulty with enrolment because of belief in the historical use of epinephrine. ${ }^{12}$ These two RCTs showed consistent increases in ROSC without evidence of a benefit in long-term survival.

Dr. Steve Lin's meta-analysis, published in 2014, concluded "the efficacy of adrenalin for OHCA remains unanswered." He and his team concluded that these findings of no statistical difference in long-term survival might have been related to insufficient power. However, even the largest sample sizes will not show a benefit if none truly exists.

The long-awaited PARAMEDIC2 trial was recently published and compared epinephrine with placebo in 8014 patients randomized by paramedics in the United Kingdom. ${ }^{10}$ Their findings echoed all the other studies 
showing greater ROSC with epinephrine, but despite a large sample size, they found no benefit in favourable neurological survival at hospital discharge or three months (modified Rankin scale [mRS] 0-3). Importantly, they found an increase in severe neurological impairment (mRS 4-5) at hospital discharge in the epinephrine group, as compared with placebo (31\% v. $17.8 \%$, respectively). This is a marker of potential harm and requires further information about the longterm neurological outcome at greater than three months. We actively await these results.

\section{Is ROSC a patient-oriented outcome?}

When PARAMEDIC2 trialists sought community input in the trial, $95 \%$ of the public chose a long-term favourable neurological outcome over short-term survival (hours to days). ${ }^{10}$ This is consistent with the inherent understanding that patients and families are not interested in surrogate outcomes but rather care whether they or their loved ones will survive and be able to care for themselves. The primary outcome of the study was 30-day survival (because of financial implications), but they did include good and poor neurological outcomes in their secondary outcomes. The results of the trial to date show no benefit in the community-chosen patientoriented outcome of favourable long-term neurological survival.

Perhaps there are other benefits associated with survival to hospital admission without long-term survival. Survival to admission may provide an easier transition for families and a chance to say goodbye to their loved ones. However, there is no direct evidence on whether this provides families with any greater comfort. The increased survival to hospital admission may benefit organ donation numbers, but there are other changes in our system and advances that could result in improved organ donation without the need to raise the hopes of families artificially and create a significant financial burden on our health care system.

If there is no benefit of epinephrine in patientoriented outcomes, why are we still using it?

With the publication of the PARAMEDIC2 study in 2018, we now have significant high-quality evidence to indicate that there is no strong rationale supporting our current (historical) recommendation of using $1 \mathrm{mg}$ of epinephrine every 3-5 minutes in all cardiac arrests. There is some evidence that an alpha-agonist medication is needed in patients who do not achieve early ROSC, but repeated doses of epinephrine appear to lead to ROSC without long-term survival and perhaps worse neurological survival.

Any future research on the use of epinephrine should focus on smaller doses, early use (before 10 minutes) in non-shockable rhythms, the use of epinephrine concomitant with beta-blocking medications, or both. It is time to think again!

AGAINST THE MOTION: EPINEPHRINE: SHORT-TERM GAIN OR LONG-TERM PAIN?

\section{Ian R Drennan (@lanR_Drennan) and Steve Lin (@emerg_doc)}

SCA continues to be an important public health issue in Canada with low survival rates. ${ }^{13,14}$ Approximately one-half of patients with SCA who are resuscitated subsequently die in the hospital as a result of neurological injuries because of prolonged ischemia during cardiac arrest and resuscitation. ${ }^{15,16}$ The International Liaison Committee on Resuscitation (ILCOR) currently suggests the routine administration of $1 \mathrm{mg}$ of epinephrine every 3-5 minutes during cardiac arrest resuscitation until ROSC or termination of resuscitation. ${ }^{17}$

The issues surrounding epinephrine administration for SCA are complex. Epinephrine has been a mainstay of cardiac arrest therapy for over 50 years. It was first introduced for the management of cardiac arrests in 1962 when it was found to increase aortic pressure in dogs. ${ }^{3}$ Epinephrine is a powerful vasopressor that increases the pressure in large blood vessels, resulting in increased aortic and coronary perfusion pressure and leading to increased rates of ROSC. ${ }^{5}$ Multiple RCTs have demonstrated that epinephrine, including higher doses, improves ROSC and survival to hospital admission. ${ }^{7,11,12,18}$

Our opponents have argued that epinephrine leads to short-term survival only and may even be harmful because of neurological injuries, and there are a number of observational studies that support this argument., ${ }^{9,19}$ This has led people to question the use of epinephrine, suggesting that we may be sacrificing neurological survival for improvements in short-term outcomes. However, observational studies only hypothesize associations, not causation, because of unmeasured (or immeasurable) confounders, particularly in cardiac 
arrest such as patient co-morbidities, duration of cardiac arrest, and cardiopulmonary resuscitation (CPR) quality, just to name a few. Perhaps, most importantly, observational studies are unable to account for the timedependent bias of epinephrine administration; patients have to be in cardiac arrest long enough to receive epinephrine, and these patients ultimately do worse because of prolonged downtimes. ${ }^{20}$

There have only been two prospective RCTs on epinephrine $\mathrm{v}$. placebo examining long-term outcomes. The first was a trial published in 2011 that was unable to demonstrate improved survival to hospital discharge or neurological function with epinephrine. ${ }^{12}$ However, the trial was hindered by lower than anticipated recruitment and was underpowered to detect a difference in either outcome. ${ }^{12}$ The recently published PARAMEDIC2 trial by Perkins et al. was a large $(>8000$ patients) RCT in the United Kingdom that evaluated epinephrine against placebo during OHCA resuscitation. ${ }^{10}$ The PARAMEDIC2 trial found that epinephrine compared with placebo significantly increased survival to discharge $(3.2 \%$ v. $2.3 \%$, respectively), at 30 days $(3.2 \%$ v. $2.4 \%$, respectively), and at three months (3.0\% v. $2.2 \%$, respectively). These numbers appear low, at first glance; however, the study population excluded patients who regained ROSC prior to any study drug representing those with very short downtimes and the highest chance of survival. The trial was not powered to detect a difference in neurological outcomes and, as expected, did not demonstrate a statistical difference in neurological outcomes (as measured by the mRS) at discharge or three months between epinephrine and placebo (discharge 2.2\% v. $1.9 \%$, respectively; three months $2.1 \%$ v. $1.6 \%$, respectively). ${ }^{10}$ Interestingly, in a subgroup analysis of survivors, the proportion of patients with a disability was higher in the epinephrine group, with $31 \%$ having a moderately severe or severe neurological impairment (mRS 4 or 5), as compared with $17.8 \%$ in the placebo group. Importantly, however, this was a secondary subgroup analysis (i.e., underpowered and a different population than that examined in the original trial design), making it difficult to draw concrete conclusions regarding the effect of epinephrine on neurological outcomes.

Immediately after publication, the results of the PARAMEDIC2 trial were met with much pessimism, with broadcasts across media claiming, "Epinephrine creates brain dead survivors." The main dilemma raised in media, social media, blogs, podcasts, etc., was which outcome is more important? Survival or survival with a good neurological outcome? Put another way: Is disability worse than death? Removing epinephrine would lead to more deaths.

\section{Should we still use epinephrine in clinical practice?}

This may not be a question that this trial can fully answer, yet the PARAMEDIC2 trial provides important evidence on the use of epinephrine in cardiac arrest. The current standard dosing of epinephrine increases survival after OHCA, but the effect on neurological disability is not clear. Although a larger percentage of survivors had poor neurological outcomes in the epinephrine group, as compared with the placebo group (31\% v. $17.8 \%$, respectively), there was an absolute increase in the number of patients with good neurological outcomes at discharge ( $87 \mathrm{v} .74$, respectively) and three months (82 v. 63, respectively), although not significant. If we take the numbers at face value, for every 1,000 patients, the use of epinephrine resulted in approximately 203 extra patients transported, 158 extra patients surviving to hospital admission, and 9 extra survivors at three months, five of whom would have good neurological outcomes.

Are five patients with good neurological outcomes worth the risks and health care costs of extra paramedic transports and intensive care unit (ICU) admissions? This is a matter of values that cannot be fully addressed by researchers or clinicians. There needs to be more public discussion and consultation on important outcomes. More specifically, when do health care resources and costs outweigh reasonable clinical outcomes and benefit? Rigorous qualitative data are needed to help address these very important and difficult questions.

\section{Knowledge gaps}

Regardless of the results of the current RCTs, removing epinephrine completely from clinical practice would be a mistake. We still know too little about the effectiveness of epinephrine despite decades of use. The PARAMEDIC2 trial provides the most definitive evidence that the current standard dosing (1 mg every 3-5 minutes) may not be ideal; maybe it is not a question of all or none? There are still knowledge gaps regarding the optimal dosage and timing of epinephrine in cardiac arrest. Observational data have shown that cardiac arrest survival decreased with higher total doses of epinephrine. ${ }^{9,21}$ The timing of epinephrine administration is 
also a major confounder. Hansen et al. showed that for every one-minute delay in epinephrine administration, there was a decrease of $4 \%$ in the odds of survival and $6 \%$ in the odds of good neurological outcome. ${ }^{22}$ Other studies have found an association between delays to epinephrine administration and decreased survival in both OHCA and in-hospital cardiac arrests. ${ }^{23-25}$ The timing of epinephrine confounds observational studies and continues to be challenging to control for in clinical trials. The mean time to epinephrine administration in the PARAMEDIC2 trial was 21 minutes after calling EMS, leaving some to suggest that the drug was provided much too late to have a positive effect on outcomes.

To help address some of the knowledge gaps in dosing, CanROC is currently planning a new RCT to evaluate a low cumulative dose of epinephrine in OHCA resuscitation. The CanROC Epinephrine Dose: Optimal versus Standard Evaluation (EpiDOSE) trial (NCT03826524) is a multicentre, double-blinded RCT across sites in Canada that will compare the current standard dosing of epinephrine to a low cumulative dose $(\max 2 \mathrm{mg})$. Patient enrolment is planned to begin in 2020.

\section{Where do we go from here?}

Epinephrine use in cardiac arrest is complicated. The PARAMEDIC2 trial has raised as many important questions as it has answered. The evidence for epinephrine is not yet definitive, one way or another. Based on one trial, if we prematurely stop using epinephrine in cardiac arrest, are we saying that disability is worse than death? Clinicians, researchers, policy-makers, and the general public need to discuss and debate our values and the evidence. If one thing is for certain, it is that with epinephrine, nothing is certain at all.

Keywords: Cardiac arrest, epinephrine, resuscitation

\section{REFERENCES}

1. Travers AH, Perkins GD, Berg RA, et al. Part 3: Adult Basic Life Support and Automated External Defibrillation: 2015 International Consensus on cardiopulmonary resuscitation and emergency cardiovascular care science with treatment recommendations. Circulation 2015;132(16 Suppl 1):S51-83.

2. Safar P. Community-wide cardiopulmonary resuscitation. f Iowa Med Soc 1964;54:629-35.

3. Pearson JW, Redding JS. Epinephrine in cardiac resuscitation. Am Heart 7 1963;66(2):210-4.
4. Attaran RR, Ewy GA. Epinephrine in resuscitation: curse or cure? Future Cardiol 2010;6(4):473-82.

5. Paradis NA, Martin GB, Rosenberg J, et al. The effect of standard- and high-dose epinephrine on coronary perfusion pressure during prolonged cardiopulmonary resuscitation. FAMA 1991;265(9):1139-44.

6. Lin S, Callaway CW, Shah PS, et al. Adrenaline for out-of-hospital cardiac arrest resuscitation: a systematic review and meta-analysis of randomized controlled trials. Resuscitation 2014;85(6):732-40.

7. Stiell IG, Hebert PC, Weitzman BN, et al. High-dose epinephrine in adult cardiac arrest. N Engl 7 Med 1992;327 (15):1045-50.

8. Perondi MB, Reis AG, Paiva EF, Nadkarni VM, Berg RA. A comparison of high-dose and standard-dose epinephrine in children with cardiac arrest. $N$ Engl 7 Med 2004; 350(17):1722-30.

9. Hagihara A, Hasegawa M, Abe T, Nagata T, Wakata Y, Miyazaki S. Prehospital epinephrine use and survival among patients with out-of-hospital cardiac arrest. $7 A M A$ 2012;307(11):1161-8.

10. Perkins GD, Ji C, Deakin CD, et al. A randomized trial of epinephrine in out-of-hospital cardiac arrest. $N$ Engl $7 \mathrm{Med}$ 2018;379(8):711-21.

11. Olasveengen TM, Sunde K, Brunborg C, et al. Intravenous drug administration during out-of-hospital cardiac arrest: a randomized trial. FAMA 2009;302(20):2222-9.

12. Jacobs IG, Finn JC, Jelinek GA, Oxer HF, Thompson PL. Effect of adrenaline on survival in out-of-hospital cardiac arrest: A randomised double-blind placebo-controlled trial. Resuscitation 2011;82(9):1138-43.

13. Nichol G, Thomas E, Callaway CW, et al. Regional variation in out-of-hospital cardiac arrest incidence and outcome. 7AMA 2008;300(12):1423-31.

14. Heart and Stroke Foundation of Canada. Position Statement on CPR: Heart and Stroke Foundation of Canada; 2016. Available at: https://resuscitation.heartandstroke.ca/guidelines/position/CPR (accessed March 17, 2019).

15. Neumar RW, Nolan JP, Adrie C, et al. Post-cardiac arrest syndrome: epidemiology, pathophysiology, treatment, and prognostication. A consensus statement from the International Liaison Committee on Resuscitation (American Heart Association, Australian and New Zealand Council on Resuscitation, European Resuscitation Council, Heart and Stroke Foundation of Canada, InterAmerican Heart Foundation, Resuscitation Council of Asia, and the Resuscitation Council of Southern Africa); the American Heart Association Emergency Cardiovascular Care Committee; the Council on Cardiovascular Surgery and Anesthesia; the Council on Cardiopulmonary, Perioperative, and Critical Care; the Council on Clinical Cardiology; and the Stroke Council. Circulation 2008;118(23):2452-83.

16. Laver S, Farrow C, Turner D, Nolan J. Mode of death after admission to an intensive care unit following cardiac arrest. Intensive Care Med 2004;30(11):2126-8.

17. Callaway CW, Soar J, Aibiki M, et al. Part 4: Advanced Life Support: 2015 International Consensus on cardiopulmonary resuscitation and emergency cardiovascular care science with 
treatment recommendations. Circulation 2015;132(16 Suppl 1):S84-145.

18. Gueugniaud PY, Mols P, Goldstein P, et al. A comparison of repeated high doses and repeated standard doses of epinephrine for cardiac arrest outside the hospital. $N$ Engl $7 \mathrm{Med}$ 1998;339(22):1595-601.

19. Behringer W, Kittler H, Sterz F, et al. Cumulative epinephrine dose during cardiopulmonary resuscitation and neurologic outcome. Ann Intern Med 1998;129 (6):450-6.

20. Andersen LW, Grossestreuer AV, Donnino MW. "Resuscitation time bias"-A unique challenge for observational cardiac arrest research. Resuscitation 2018;125:79-82.

21. Glover BM, Brown SP, Morrison L, et al. Wide variability in drug use in out-of-hospital cardiac arrest: a report from the resuscitation outcomes consortium. Resuscitation 2012;83 (11):1324-30.
22. Hansen M, Schmicker RH, Newgard CD, et al. Time to epinephrine administration and survival from nonshockable out-of-hospital cardiac arrest among children and adults. Circulation 2018;137(19):2032-40.

23. Nakahara S, Tomio J, Nishida M, et al. Association between timing of epinephrine administration and intact neurologic survival following out-of-hospital cardiac arrest in Japan: a population-based prospective observational study. Acad Emerg Med 2012;19(7):782-92.

24. Tanaka H, Takyu H, Sagisaka R, et al. Favorable neurological outcomes by early epinephrine administration within 19 minutes after EMS call for out-of-hospital cardiac arrest patients. Am 7 Emerg Med 2016;34(12):2284-90.

25. Andersen LW, Kurth T, Chase M, et al. Early administration of epinephrine (adrenaline) in patients with cardiac arrest with initial shockable rhythm in hospital: propensity score matched analysis. BM7 2016;353:i1577. 